\title{
Audit officers: what are they up to?
}

\author{
Jenny Firth-Cozens, Pamela Venning
}

Audit assistants, audit officers or coordinators, research officers, facilitators - it seems that the range of titles of those people whose task should be purely audit is bettered only by the large differences in their job descriptions and the actual activity they find themselves undertaking. This article outlines some of the variety of their backgrounds and experiences in their new roles, roles which some see as crucial to the success of the medical audit programme.

\section{Where do audit officers come from?}

It is fascinating to witness, emerging from such a diversity of backgrounds, a new professional group in which there is already enough feeling of cohesion to encourage it to form its own professional society. Audit officers have come together from several occupations within the NHS-psychology, medical records, nursing, secretarial, physiotherapy, laboratory, and information services-and from some areas outside the health service, such as computing, commerce, and academia. Their backgrounds seem perhaps less important than their ability to adapt and to think logically, to deal tactfully and helpfully with groups of consultants, to enjoy data and their analysis and presentation, and to get things done. Some people do feel, however, that at least some previous steeping in NHS culture is useful, especially if it has provided a knowledge of clinical terms.

\section{Role and rewards}

Our experience is that a good audit officer can and should take part in every stage of the audit cycle. Officers should be present at the design so that they can facilitate discussion on the question being asked and on the purpose of the audit and point out (more usefully for being non-clinicians) the need for precision in definitions and standards: "How will $\mathrm{I}$, as a nonclinician, know that this has been achieved?" is a vital question if data are to be in any way objective. They should agree how data will be collected-by themselves or by a clerk or other professional-and they should analyse the data and present the results back to the specialty group. If officers find some aspects of these activities difficult then these should be addressed in training: the extra funding provided for establishing audit this year cannot be better spent than in providing training for a group that has been, in some places, expected to metamorphose into experts overnight.

This is the average role, the post most commonly called audit officer or coordinator, for which the salary is around $£ 14000$ on scale 5. Below these posts are those of audit clerks, who are often on scale 3 , who enter and perhaps collect data and provide the simplest of analyses. Above audit officers or coordinators are rarer individuals, usually called audit facilitators: those who coordinate audit activity across the district, who are on senior management salaries of $£ 18000$ and above. There are still large anomalies in job descriptions and the salaries that accompany them, and it will be a task of the newly formed society to try to reduce such variation.

If audit activity is well staffed and well managed then audit officers will take over most of the burdens of clinicians, who will be able to spend their time on design of audit, setting standards, and initiating changes highlighted as being necessary. This is why it is so important that audit officers are not side tracked (whether by clinicians or by management) into other activities such as writing computer programs, having to pull patients' notes, secretarial work, data input, or research. In this regard, it is worth deciding who pulls the notes before the appointment so that the new officer isn't faced with irate or intractable medical records staff, as we know has happened frequently.

In some regions a regional audit coordinator has been appointed, again with several roles. One of us (JF-C) has a background in research, clinical psychology, and organisational development, and her role is the facilitation of audit-getting it goinglargely through workshops, evaluation of change, and coordinating medical audit with the introduction of audit by other professional groups. The other (PV) has a background in nursing and audit, and her role is providing expertise to those undertaking regional audits and providing training for audit officers throughout the district. How these posts are defined will depend very much on local need and know how.

\section{What do audit officers need?}

Even in regions in which the appointment of audit officers was deferred until there was clearly a role for them, we still hear of first days in the job with nothing to do, no office, no clear relationship with anyone.

\section{There is often a temporariness about their posts which must be hard to take...}

There is often a temporariness about their posts which must be hard to take: accommodation in portacabins, no office cleaning, refusals to supply stationery, no recognition by the switchboard, or exclusion from the circulation list. This is largely because many officers are on short term contracts and all are funded with ringfenced money, which seems to give them an ephemeral quality. It certainly does seem to fulfil the intentions of the Department of Health that audit is here to stay and that its funding will eventually be provided for through contracts.

Most audit staff relate to the chairperson of the district audit committee (or its equivalent since 1 April) for their work and to someone in management, information, or quality for their working conditions. The fewer the audit officers employed, the more support the chairperson should provide: the job can be lonely and, with its newness, it can be both vague and difficult. Again, we have heard of great variation in this relationship: from officers who rarely meet the chairperson, except at formal meetings, to a chairman who hangs his coat in the person's office and does most of his audit activity from there. Certainly chairpersons will find that they retain these valuable audit staff more 
easily if they put their weight behind getting such staff decent accommodation and ensuring that their duties are regarded as a part of the hospital's day to day activity.

Now that money has been given to regions separately for the inception of clinical audit - by nurses and other professional groups-it seems important that audit officers should be used more widely, when appropriate, rather than separate audit empires being established, which will be detrimental to audit itself and to the staff involved. Where local clinical audit committees are being established, consisting of medical and other professionals, it seems less likely that this will occur and this generally must be a more fruitful progression.

\section{Training needs}

One question which should be addressed is that of who will determine the training needs of audit officers. Ideally, of course, the officers themselves should identify these needs to the chairperson or the district audit committee. This, however, requires establishing a culture in which asking for training is seen as a positive action rather than a deficiency.
Many courses are springing up around the country, providing national training on topics both broad and narrow, and feedback on their quality varies wildly. A different approach, which might in the long term prove more fruitful, is where regions have set up local initiatives which, after an introductory course, aim at allowing the participants to design their own future syllabus according to their particular development needs. From such a programme we have seen requests for less predictable audit topics such as assertiveness training, medical terminology, and budgeting skills for those who find themselves involved in handling financial aspects of the job.

\section{Conclusion}

Audit, whether medical or clinical, is here to stay, our experience, young as it is, already suggests that audit flourishes best when well motivated and wel cared for audit staff are employed. The more fully they are allowed to participate in the audit activity the better motivated they will be, and the more integrated they are into the hospital or community setting the more likely it is that they will stay and make audit work.

\section{DIARY}

\section{September}

London: St George's Hospital. Provision of treatment guidelines in NHS hospitals (fee $\{5$ (by Sept 16)) Contact Dr Ina Ismail, Department of Clinical Pharmacology, St George's Hospita Medical School, London SW17 0R

\section{September-Decembe}

Scotland: West of Scotland Committe for Postgraduate Medical Education. Introduction to computing for medical audit. One day courses, each limited to eight or 10 participants, for all doctor working in the six West of Scotland health boards. Contact Dr Sue Kinn, Glasgow University (tel 0413398855 ext 5611).

\section{October}

London: Hotel Russell. Preliminary meeting to constitute a medical audit association. (Audit staff not already notified contact Patricia Kent, King's Fund Centre (ext 205).)

\section{1-13 October}

Harrogate: National Health Service Training and Studies Centre. A thre day basic course in medical audit for audit assistants and support staff. Contact Cynthia Commons (tel 0472210908 ) or Patricia Kent (tel 0712673800 ).

\section{1-12 October}

Brighton: Brighton General Hospital. Organisational structure and system for planning and evaluating medical audit (fee £325). Contact Mr Mark Renshaw, Brighton General Hospital, Elm Grove, Brighton BN2 3EW (tel 0273696011 ext 3971; fax 0273697671).

Items for possible inclusion in the news section to the manager, Medical Audit Programme King's Fund Centre, 126 Albert Street, London NWI 7NF (tel 071267 6111; fax $0712676108)$.

\section{Audit Views}

I troduction of a revised policy for closer monitoring of intravenous antibiotic use led to a $23 \%$ fall in costs in the first four months without compromising patient care (American fournal of Medicine 1991;90:439-44). Interventions in $42 \%$ of cases resulted in lower doses, longer intervals between them, and shorter treatment. The extra workload was offset by appointing a pharmacist to the infectious diseases team - at a salary equivalent to half the savings achieved.

A dit of five years' vascular surgery by a surgeon enthusiast showed that outcome was satisfactory except for below knee bypass operations and a high rate of reoperation for postoperative bleeding (British fournal of Surgery 1991;78:601-6). Comparison with other surgeons' experience proved difficult because most published work comes from abroad and statistics in Britain are inadequate. Perhaps British vascular surgeons should collaborate to produce them.

$\mathrm{A}$ experienced clinician may be able to predict the outcome of weaning a patient from a ventilator, bu objective indices would be valuable. The best index seems to be rapid shallow breathing, quantified by the ratio of respiratory rate to tidal volume in the first minute after removal of the ventilator (New England Fournal of Medicine $1991 ; 324: 1446-50)$. It is simple to measure; its sensitivity and specificity are high; and a threshold value of 100 , above which weaning is unlikely, is easy to remember.

\section{$\mathrm{S}$} uart Logan (Archives of Disease in Childhood 1991;66: 745-8) rejects most outcome measures recommended by paediatricians to evaluate services because they are commonly concerned with social rather than medical interventions; besides, adverse outcomes in children are, fortunately, rare, so very large numbers would be needed to show changes. He would prefer to see policy evaluation separated from medical audit, which requires its own research studies, as well as close discussion of each adverse outcome.

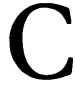
nicians' complaints about slowness in receiving histopathology reports might be minimised if laboratories adopted an "achievement index" (Fournal of Clinical Pathology 1991;44:492-6), based on turnround time of speci- mens and the requirements of an arbitrary $80 \%$ of clinicians Computerisation should make it possible to satisfy individual "required by" dates-for example, in cases of suspected malignancies and biopsies in inpatients.

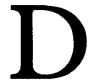
ring the six months after establishing an asthma clinic run by a nurse in general practice doctor consultations were nearly halved, oral corticosteroid treatment fell by over a half, and the need for emergency salbutamol was reduced by two thirds (British fournal of General Practice 1991;41:27-31). Eighty per cent of patients had their treatment modified, and there were reductions in out of hours consultations and home visits.

A nother study of asthma concerning audit of minimal intervention using disability scores in a two doctor practice in Shropshire showed improvements in patients' knowledge about the disease and inhaler technique and their greater use of peak flow meters (British fournal of General Practice 1991;41:232-6). Although management was thought to be generally satisfactory, only $44 \%$ of patients were regularly supervised, and the value of a special clinic is to be assessed in the next audit.

A though rates of necropsies in neonates are relatively high compared with those in adults, nearly half the neonatal necropsies carried out in the West Midlands failed to attain even minimum agreed procedural standards (British Fournal of Obstetrics and Gynaecology 1991;98:624-7). Parents whose future choices may depend on the result of the necropsy are entitled to expect high quality, and managers may complain that a cost of up to $£ 1000$ a time hardly represents value for money.

$\mathrm{A}$ small and often isolated specialty like ear, nose, and throat surgery may be best audited on a regional basis, but an individual unit can provide a varied programme which generates an enthusiastic response. A successful example is described (Fournal of Laryngology and Otology 1991;105:611-3) in which hourly meetings each week are allocated to consideration of workload in one week's meeting, morbidity and mortality the second, managerial problems in the third, and topics such as technical procedures, specific complications, diagnosis, and treatment in the fourth. 